\title{
Basics of Neurointervention
}

\author{
Tariq Matin ${ }^{1} \quad$ Aviraj Deshmukh² A. R. Al-Schameri ${ }^{3}$ \\ ${ }^{1}$ Department of Neurointervention, Narayana Super Speciality \\ Hospital, Gurugram, Haryana, India \\ ${ }^{2}$ Research Institute of Neurointervention, Christian Doppler Clinic, \\ Paracelsus Medical University, Salzburg, Austria \\ ${ }^{3}$ Department of Neurosurgery, Christian Doppler Clinic, Paracelsus \\ Medical University, Salzburg, Austria \\ Indian J Neurosurg 2018;7:96-101
}

\begin{abstract}
Address for correspondence Tariq Matin, MBBS, DMRD, DNB, Department of Neurointervention, Narayana Super Speciality Hospital, Nathupur, Sector 24, Gurugram, Haryana, India122002 (e-mail: tariqmatin@yahoo.co.in).
\end{abstract}

\begin{abstract}
Keywords

- neurointervention

- catheter

- wires

Neurointervention is a subspecialty of neurosciences which offers a minimally invasive therapy for the vascular lesions of the central nervous system. The resources, techniques, and indications of neurointervention therapy are rapidly expanding and becoming diverse. With the support of robust scientific data, neurointervention has established itself as a first line therapy for many neurovascular diseases. This article provides an overview about the various material used in neurointervention, basic steps followed in some common neurointerventional procedures, and its related complications. In the era of 21st century, any young physician or surgeon as well as residents-in-training dealing with neurovascular cases should have some insight into these basic concepts so as to provide better care to their patients.
\end{abstract}

\section{Introduction}

Neurointervention offers minimally invasive therapies for a wide variety of neurovascular diseases. Over the years it has evolved and established itself as a preferred modality of treatment over conventional open surgical approach. Adoption of endovascular therapy for the management of aneurysms, acute stroke, vascular malformations, presurgical, or palliative embolization has expanded over last three decades. This trend is likely to amplify further with the introduction of innovative endovascular devices. Neurointervention spans across various specialties, namely: neurosurgery, neuroradiology, neurology, and cardiology. Accordingly, it has embraced many names such as endovascular neurosurgery, interventional neuroradiology, and interventional neurology. The aim of this review is to provide basic idea about indications and technical concepts in neurointervention, which is becoming prerequisite for neurology, neurosurgery, as well as radiology residents training in this era.

\section{Historical Aspect}

Of the endovascular therapies, embolization of aneurysm is one of the principal procedures. Although diagnostic cerebral angiography was developed by Egaz Moniz in 1927, the

received

July 7, 2018

accepted after revision

July 9,2018

published online

September 6, 2018 endovascular approach to aneurysm treatment took many years to develop. In 1964, Luessenhop and Velasquez performed the first catheterization of intracranial vessels. ${ }^{1}$ Subsequently, in 1974, the description of use of detachable and nondetachable balloons by Serbinenko in his series of 300 patients started the balloon era of endovascular treatment of intra-arterial. ${ }^{2}$ The rigid structure of balloon produced an angioplasty of the aneurysm wall with consequent immediate or delayed aneurysm rupture. Hence, their use remained limited to the small clinical series of inoperable aneurysms. Development and use of electrolytically detachable platinum coils (Guglielmi detachable coil [GDC]) in 1991 by Guglielmi et al initiated the modern era of neuroendovascular therapy. ${ }^{3}$ With continued advances in endovascular technology, aneurysm coiling rapidly became an accepted and viable alternative to surgical clipping over the last decade of the 20th century. Similarly, endovascular intervention is now a standard of care for acute treatment of large vessel stroke. ${ }^{4}$ Availability of newer embolic agents had made treatment of vascular malformation easily amenable. Apart from newer devices, improvement in catheters and guidewire technology with improved trackability, better proximal and distal support, availability of newer antiplatelet agents, widespread availability of cross-sectional imaging, and neurocritical care facility have improved outcomes following endovascular treatment.

(C2018 Neurological Surgeons' Society of India
License terms

()(1) $\Theta \circledast$ 10.1055/s-0038-1670934 ISSN 2277-954X. 


\section{Indications of Neurointerventional Therapy}

As mentioned above, the important indications of endovascular therapy are:

1. Aneurysm treatment: both ruptured as well as unruptured aneurysms.

2. Large vessel stroke: Class A, Level I evidence (American Heart Association guidelines).

3. Vascular malformations: arteriovenous malformations (AVMs), dural fistulas, and venous anomalies.

4. Preoperative tumor embolization.

5. Others: epistaxis, vertebroplasty, kyphoplasty, etc.

\section{History Taking and Neurological Examination}

Before performing any diagnostic of therapeutic endovascular procedure, it is important to examine old records, imaging findings, and aim of the therapeutic procedure. A focused neurological examination is pivotal before any procedure. Adequate planning including choice of catheters and wires to be used should be made beforehand according to the neurovascular anatomy, availability of resources, and operator preference. An informed consent explaining the calculated risks associated with the concerned procedure is very important to avoid medicolegal complications afterwards.

\section{Devices, Drugs, and Material Used in Neurointervention Contrast Material}

Nonionic contrast agents are used in neurointervention procedure. Judicious use of contrast is advisable to avoid any contrast-related adverse effects, including allergic reactions and nephrotoxicity. Adequate hydration and avoidance of nephrotoxic drugs should be part of preprocedural planning. Certain protocols should be followed in neurointerventional cath lab to limit the total dose of contrast used such as using lowest-strength contrast whenever possible, using contrast syringes on the table filled half-and-half with contrast and saline, elimination of unnecessary runs, careful planning of injection, and fields of view to avoid need for repeated injections.

\section{Periprocedural Anticoagulation with Heparin}

It is important to remember that the majority of the complications during a neurointerventional procedure is thromboembolic in nature and hence perioperative anticoagulation therapy is vital. All patients undergoing interventions should be anticoagulated with heparin except if contraindicated. The initial bolus dose is approximately 60 to $80 \mathrm{U} / \mathrm{kg}$ followed by 20 to $40 \mathrm{U} / \mathrm{kg}$ every hour, with an aim to maintain activated partial thromboplastin time of 2 to 2.5 times the baseline value. The only exception to the use of heparin is in subarachnoid hemorrhage where anticoagulation is deferred until one or two coils are deployed to secure the aneurysm. All the catheters and sheaths should also be continuously flushed with heparinized saline $(1,000-2,000 \mathrm{U}$ in $1 \mathrm{~L})$ with the help of pressure bags as per the institutional protocol. ${ }^{5}$

\section{Use of Antiplatelets}

Oral as well as intravenous antiplatelet agents are used in neurointerventional surgery to prevent thromboembolism during and after the procedures. Use of dual antiplatelets is necessary when adjuvant devices such as flow diverters, stents, or neck or neck bridge devices are used. In most of the centers, dual antiplatelets are continued for a period of up to 1 year followed by single antiplatelet indefinitely. Aspirin, clopidogrel, prasugrel, and ticagrelor are the routinely used oral antiplatelet agents. Recently, various platelet function tests have become available in the market to assess the effect of oral antiplatelet agents. Their use is still limited due to lack of standardization. ${ }^{6}$

Intravenous antiplatelet agents are used when rapid effect is required such as stent thrombosis or thromboembolic complications. Abciximab, integrilin, eptifibatide, and tirofiban are the common intravenous (IV) antiplatelet agents available for clinical use. Indications and side effects of both oral as well as parenteral vary, therefore selection of the appropriate antiplatelet agent depends upon the patientrelated factors. ${ }^{7}$

\section{Catheters}

Various catheters are used for diagnostic and therapeutic procedures. They vary in their size, shape, length, trackability, radiopacity, and transition zones according to their intended use. Various catheter types include diagnostic catheters, guiding catheters, distal access catheters, and microcatheters. Diagnostic catheters are usually 90 to 100 $\mathrm{cm}$ in length, $4 \mathrm{~F}$ to $6 \mathrm{~F}$ in size, and available in various tip shapes such as straight tip, angled, Simmons (1, 2, and 3), Osborn (1 and 2), Headhunter type, etc. (-Fig. 1). Spinal diagnostic catheters are smaller in length $(65 \mathrm{~cm})$ and are also available in different shape as well, for example, Cobra catheter. Longer length diagnostic catheters are used when there is need of coaxial support for the advancement of guide or distal access catheters.

Guide catheters with wide lumen and long length catheters are used in therapeutic procedures and are available in $6 \mathrm{~F}$ to $8 \mathrm{~F}$ size and 110 to $135 \mathrm{~cm}$ length. Their aim is to provide access to large vessels and support to microcatheter which is used to reach to the site of interest in cerebral vasculature. Procedures can easily fail or be unnecessarily prolonged because of poor guide catheter selection or compromised catheter position. ${ }^{8}$ Recent addition to the system of guide catheters is the use of balloon guide catheters (BGCs) in

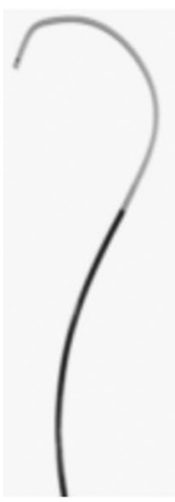

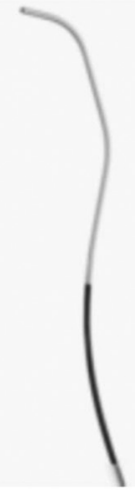

b

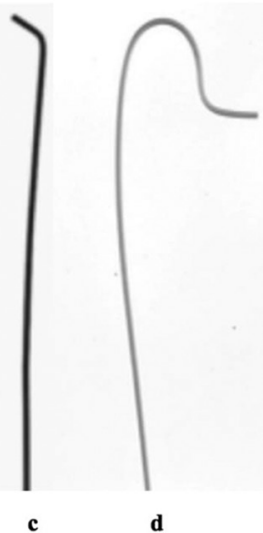

Fig. 1 Diagnostic catheters. From left to right: (A) Cobra, (B) Headhunter, (C) Angled/Vertebral, and (D) Simmons. 
stroke, which allow proximal occlusion and thereby prevent distal embolization as well as improve rates of recanalization. ${ }^{9}$ Distal access catheter is a newly developed set of catheters with extreme navigability and trackability intended to give extra support in neurointervention cases. The larger lumen distal access catheters are also used as aspiration catheters for the treatment of stroke, due to their easy and rapid navigation characteristics. ${ }^{10,11}$

On the other hand, microcatheters are soft and floppy as they are designed to be least traumatic. Once stable position is achieved with a guide catheter or distal access catheter, microcatheters are then advanced to the exact site in the cerebral vasculature. They have different lengths, diameters, stiffness, tractability, radio-opaque markers, and some of them are even detachable for use in vascular malformations. Selection of appropriate microcatheter depends on the position of the guide catheter, microcatheter flexibility, stability, and intended use of such coil deployment, liquid embolization, stent deployment, etc. Ideally, we want a catheter that can be pushed and tracked and yet remain stable.

\section{Wires}

Similar to the catheter system, various wires are used in diagnostic and therapeutic procedures. For diagnostic procedure, most commonly, hydrophilic 0.035 -inch angled guidewire is used, while stiff guidewires (0.038 inch) are used in the presence of a tortuous anatomy. Nonhydrophilic guidewires may be used to exchange catheters due to their better stability. Microwires have a diameter ranging from 0.007 to 0.021 inch. They are used to navigate microcatheters, balloons, stents, and other devices intracranially. ${ }^{11}$ An appropriate selection of microcatheter and microwire are cornerstone in successful treatment of neurovascular diseases. Selection, uses, various shaping methods, and techniques of these devices are beyond the scope of this review.

The interventionalist can choose from a variety of available catheters and guidewires. The choice of material depends on the intended use, neurovascular anatomy, availability, and most importantly, preference of the neurointerventionist. Proper selection of materials can be learned only through experience and hands-on training. The usual setup for the therapeutic procedure involves short sheath/ long sheath, guiding catheter, microcatheter, and microwire (-Fig. 2). Before beginning a procedure, one should ensure compatible of the selected devices with one another. Dry table test is sometimes necessary if there are any doubts or if using unfamiliar equipment. Always remove the slack from the system. Begin slowly and proceed gently. If something is not advancing as expected, understand why. Do not simply push hard!

\section{Embolic Agents}

Therapeutic or preoperative embolization is an established treatment of many head and neck and intracranial vascular lesions such as AVMs, fistulas, and hypervascular tumors. Embolic agents are basically divided into two groups according to their duration of action, that is, temporary and permanent ( - Fig. $\mathbf{3}$ ). Choice depends on the desired clinical outcome, as well as the inherent properties and behavior of the agent. The temporary agents include gelfoam, collagen, and thrombin. Permanent agents include particles (polyvinyl alcohol [PVA]), coils (pushable, injectable, and detachable), liquid agents (glue, alcohol, and onyx), and some miscellaneous agents (Amplatzer plug and detachable balloons). ${ }^{12}$
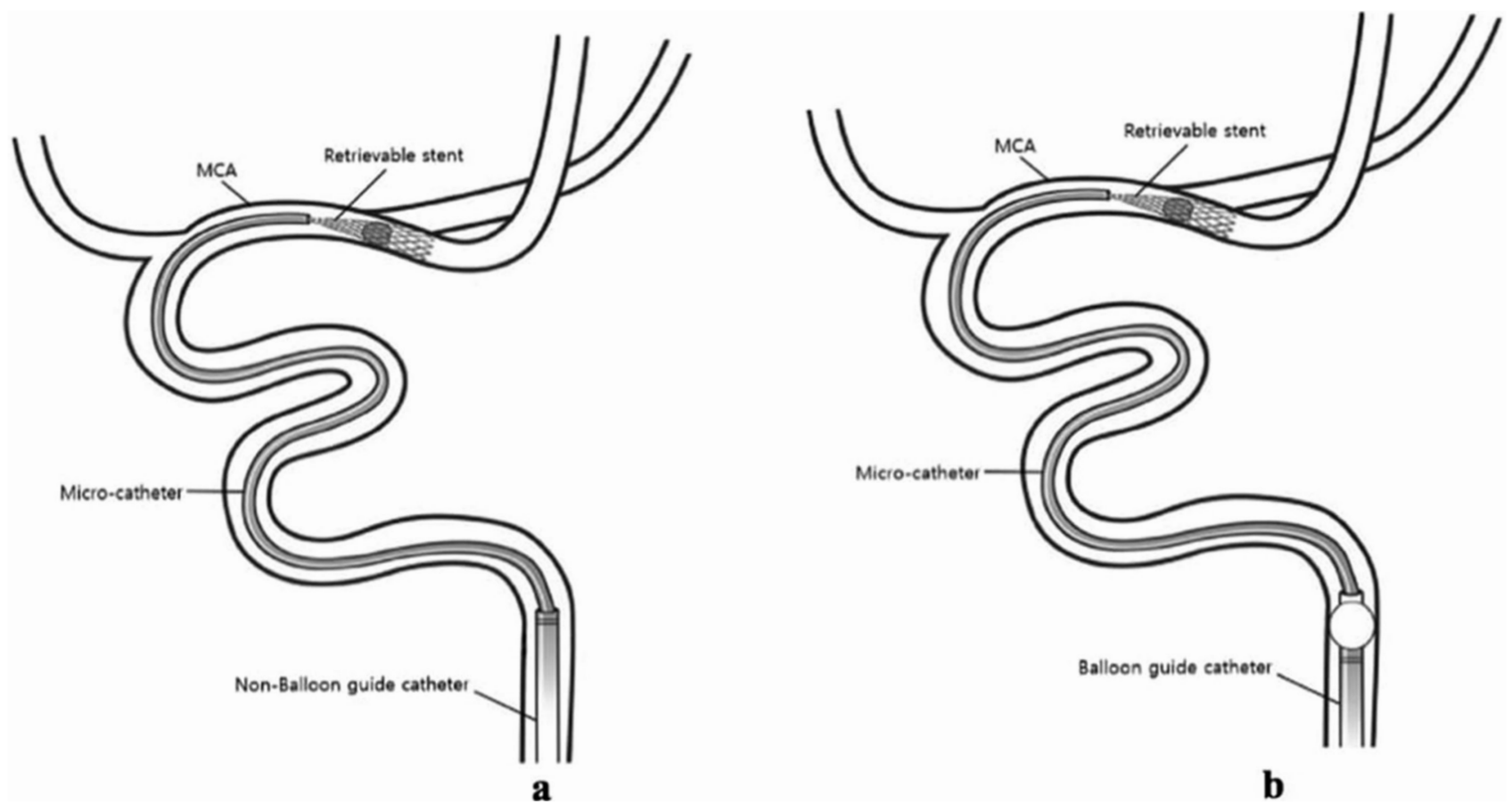

Fig. 2 Setup for stent retriever-based mechanical thrombectomy without (A) and with balloon guide catheters (B). 


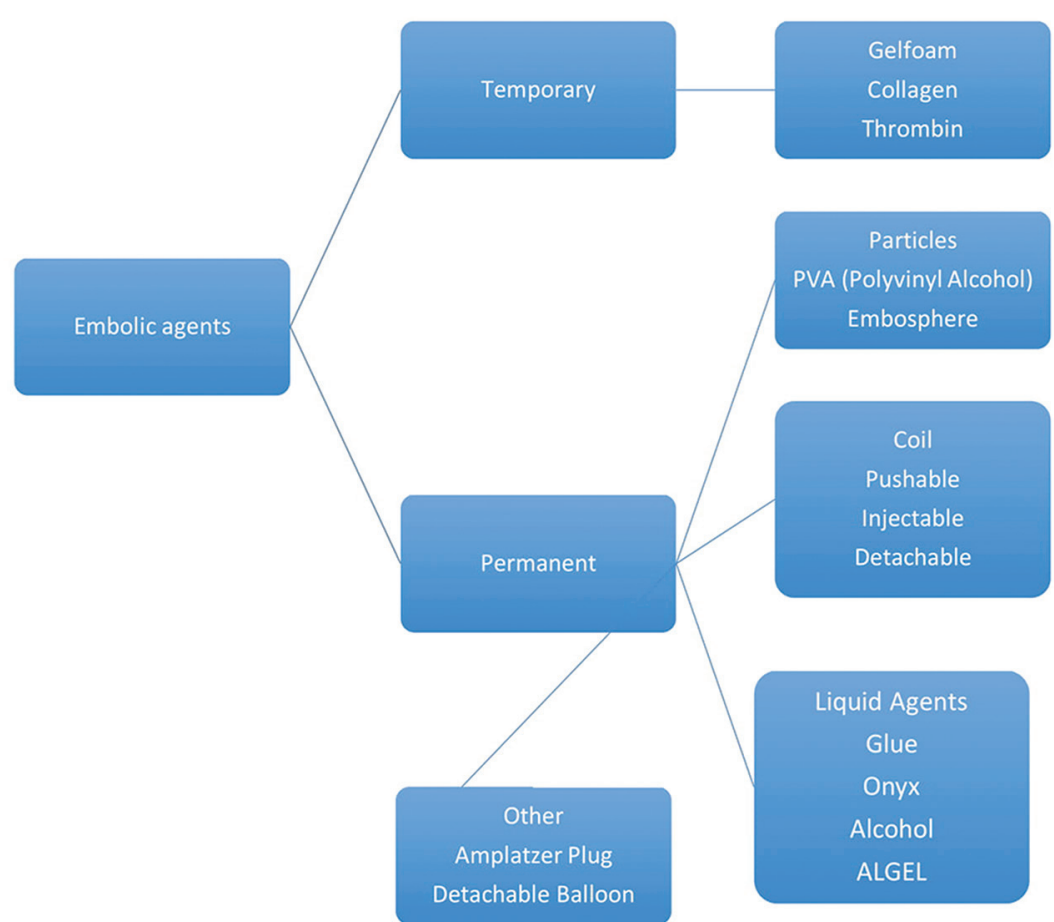

Fig. 3 Classification of embolic agents.

\section{Basic Steps in Diagnostic and Therapeutic Neurointerventional Procedures Arterial Access}

Apart from special circumstance, arterial access is always obtained via the common femoral artery (CFA). The CFA crosses from the abdomen into the thigh at midpoint of the inguinal ligament. The angle of insertion of the arteriotomy needle is approximately $45^{\circ}$ to the skin and at the site that allows compression against a bone afterward. Seldinger technique with a 0.035-inch wire is a standard approach (-Fig. 4). Commonly, a $5 \mathrm{~F}$ sheath is used for diagnostic procedures in adults and a $4 \mathrm{~F}$ one in children. For interventional procedures, a larger sized sheath is required and, therefore, its size may vary from $6 \mathrm{~F}$ to $9 \mathrm{~F}$. Length of the sheath varies from 10 to $90 \mathrm{~cm}$. The radial artery, brachial artery approach, and the direct carotid artery puncture are used when the femoral arterial access is not available or when the great vessels in the neck are tortuous enough to preclude a safe catheterization. The transradial approach is gradually increasing as an alternative approach for posterior circulation lesions to avoid navigation through the aortic arch to catheterize the vertebral artery. ${ }^{13}$

\section{Aneurysm Coiling}

As already mentioned, the usual set up for the aneurysm coiling is placement of short sheath/long sheath, guiding catheter, and microcatheter. Once a microcatheter is placed at the appropriate site, the choice of material and/or devices used for coil embolization differ according to the aneurysm sac morphology, clinical scenario, and availability of health care resources, including skill and experience of the operating interventionists. With the development of GDC coil in

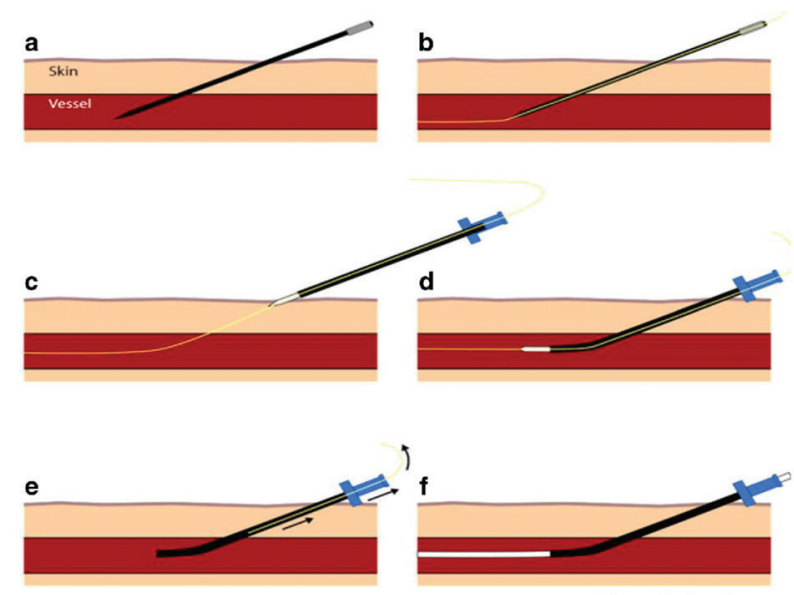

Fig. 4 After placing a hollow-core needle into the artery (A), a guidewire is inserted through the needle and advanced into the artery (B). The needle is exchanged for a sheath $(\mathbf{C}-\mathbf{E})$ and then the wire is removed $(\mathbf{F})$.

1990, it remained the preferred treatment of choice for the next decades. ${ }^{3}$ Simple unassisted coiling is usually suitable for IA with a favorable aneurysmal anatomy in terms of neck width, dome-to-neck ratio, aspect ratio, and relationship with the branch vessels. In the treatment of complex aneurysms with unfavorable dome-to-neck, aspect score, or wide neck, it is difficult to achieve coil stability with an unassisted coiling technique. More complex aneurysms with unfavorable aneurysmal anatomy can be treated with adjunct techniques, where additional support, in the form of a balloon or stent, is used to provide necessary scaffolding (- Fig. 5). Both of these devices allow denser coil packing when compared 


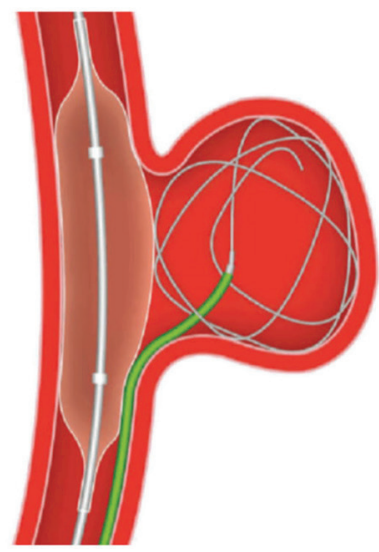

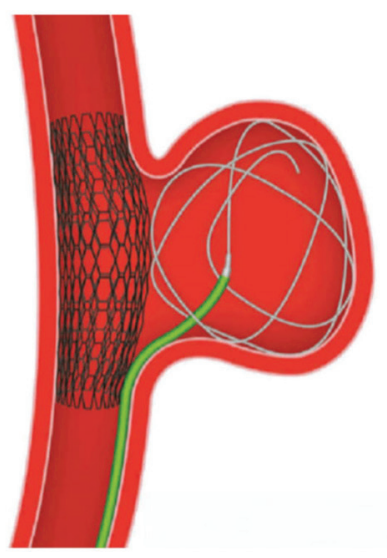

Fig. 5 Use of balloon and stent in the treatment of wide neck aneurysms.

with conventional unassisted coiling. ${ }^{14} \mathrm{~A}$ variety of balloons including compliant, hypercompliant, round-shaped, and double-lumen balloons are available. Stent-assisted coiling is valuable when there is extremely unfavorable dometo-neck ratio $(<1.0)$ and where permanent support is required to maintain coil position. Another indication for stenting is in the treatment of dissecting aneurysms. Single or overlapping stents are used to trap the dissection flap and close the tear, thereby restoring wall integrity. ${ }^{15}$ Flow diverters (FDs), which are based upon the principle of modifying intra-aneurysmal hemodynamics, are one of the most recent and substantial additions to the armamentarium for aneurysm treatment. The design of flow diverting stents is similar to conventional stents but with much lower porosity. FDs provide a scaffold for endothelial growth, thereby isolating the aneurysm from the parent circulation. ${ }^{16}$ Several novel devices which are still in various stages of development and trials are forthcoming, which include intrasaccular flow disruptors and neck bridge devices such as pCONus Bifurcating Aneurysm Implant (Phenox) and PulseRider (Pulsar Vascular).

\section{Mechanical Thrombectomy}

Endovascular intervention is an established treatment for acute treatment of stroke with a Class A, Level I evidence. ${ }^{4}$ Though currently recommended for up to 6 hours, evidence is now coming up that might make endovascular stroke therapy possible in selected patients even up to 24 hours after symptom onset. ${ }^{17}$ The two treatment strategies are the stent retriever-based thrombectomy or aspiration thrombectomy, popularly called as ADAPT. ${ }^{10}$ These treatment options can be used alone or can be combined together and is known as a Solumbra technique. ${ }^{18}$ Various stent retrievers as well as aspiration catheters are available for clinical use, with unique advantages and disadvantages. The usual working set up for mechanical thrombectomy is the use of either $6 \mathrm{~F}$ to $8 \mathrm{~F}$ long sheath or a large bore ( $8 \mathrm{~F}$ or $9 \mathrm{~F}) \mathrm{BGC}$ ( - Fig. 2 ). In stent retriever-based thrombectomy, microcatheter along with microwire is then advanced across the site of occlusion, while in aspiration thrombectomy a $5 \mathrm{~F}$ or $6 \mathrm{~F}$ aspiration catheter is advanced just proximal to the thrombus. With the availability of microthrombectomy stents and small-caliber aspiration catheters, it is now possible to offer endovascular treatment in selected group of patients with distal intracranial vessel occlusion when there is failure or contraindication to IV recombinant tissue plasminogen activator. ${ }^{19}$

\section{Treatment of Vascular Malformations, Preoperative Embolization}

Availability of newer embolic agents has made successful partial or complete treatment of various vascular malformations such as AVM and dural arteriovenous fistula. Liquid glue was a preferred agent for such treatment till the discovery of onyx. Liquid glue polymerizes into a solid material on contact with blood or tissue. Onyx (ev3/Covidien) is a copolymer of ethyl vinyl alcohol prepared with dimethyl sulfoxide (DMSO) as the solvent.

Tantalum powder is added to make the mixture radio-opaque. Three varieties are available depending upon the viscosity: Onyx-18, Onyx-34, and Onyx-500. ${ }^{20}$ Once it comes in contact with blood, it forms a case within the blood vessels. Although glue can be delivered through any microcatheter, a special DMSO compatible microcatheter is necessary for onyx injections.

Preoperative tumor embolization is becoming a standard of care for the treatment of vascular tumors to avoid excessive hemorrhage. Agents such as gelfoam, PVA, or glue are preferred for preoperative embolization.

\section{Vascular Closure Device}

Manual compression of the CFA against the head of the femur has been the traditional way of achieving hemostasis after an arterial puncture. However, it causes discomfort to the patient, causes a delay in achieving hemostasis, and limb mobilization. Closure devices aim to achieve hemostasis while reducing patient discomfort and saving time and effort. The most commonly used closure devices in neurovascular interventions are Angioseal (St. Jude Medical Inc.), StarClose (Abbott Vascular), and Perclose (Abbott Vascular). The Angioseal is available in $6 \mathrm{~F}$ and $8 \mathrm{~F}$ sizes and seals the arteriotomy site by sandwiching the site between an intraluminal anchor and a collagen plug on the external surface. All the components are reabsorbed within 60 to 90 days. The StarClose device is approved for $<6 \mathrm{~F}$ arteriotomies and achieves hemostasis using a 4-mm nitinol clip which grasps the arteriotomy edges and pulls the vessel walls together. Perclose is a suture-based device. A single device can seal $8 \mathrm{~F}$ arteriotomies, while multiple devices can be combined together to even seal larger arteriortomies. ${ }^{21}$

\section{Complications Related to Endovascular Treatment}

Every step of neurointerventional procedure-diagnostic as well as therapeutic-carries risk and it varies according to the clinical case scenario, experience of the neurointerventionist, and patient-related factors. Risk of permanent neurological 
deficit for diagnostic angiography is 0 to $0.5 \%$, while it increases up to $10 \%$ for complex aneurysm coiling. ${ }^{22}$ The most important complications are thrombotic and thromboembolic in nature. Others include intracranial hemorrhage, subarachnoid hemorrhage, arterial dissection, access site complications, vasospasm, and device failure. Risk is more in cases where additional devices such as balloon or stent are used. Similarly, FDs are a feasible and effective tool for the treatment of complex aneurysms, but have a unique risk of intraparenchymal hemorrhage and delayed aneurysm rupture. ${ }^{23}$ Apart from routine complications of neurointerventional procedures, mechanical thrombectomy has its unique set of complications, inherent to the procedure including recanalization failure, reperfusion hemorrhage, and embolization to previously unaffected territories. One should also remember the risks associated with radiation exposure, particularly when dealing with susceptible population such as children. Both neurointerventionists and neurocritical care team should be aware of the risk factors, strategies for prevention, and management of these complications.

To summarize, endovascular treatment is a minimally invasive, exciting, and therefore rapidly expanding endovascular specialty. A wide range of complex neurovascular disorders are now treatable with new endovascular technology. Although the knowledge of different devices, techniques, and neuroanatomy is important, another critical piece of the puzzle is your work environment. Effort put into building networks of support repay enormously, particularly when procedure-related complication arises. A young aspiring resident should be aware of the challenges of the specialty and prepare oneself to handle them with confidence and courage.

\section{Conflict of Interest}

None.

\section{References}

1 Luessenhop AJ, Velasquez AC. Observations on the tolerance of the intracranial arteries to catheterization. J Neurosurg 1964;21:85-91

2 Serbinenko FA. Balloon catheterization and occlusion of major cerebral vessels. J Neurosurg 1974;41(2):125-145

3 Guglielmi G, Viñuela F, Sepetka I, Macellari V. Electrothrombosis of saccular aneurysms via endovascular approach. Part 1: electrochemical basis, technique, and experimental results. J Neurosurg 1991;75(1):1-7

4 Powers WJ, Derdeyn CP, Biller J, et al; American Heart Association Stroke Council. 2015 American Heart Association/American Stroke Association Focused Update of the 2013 Guidelines for the Early Management of Patients With Acute Ischemic Stroke Regarding Endovascular Treatment: a guideline for healthcare professionals from the American Heart Association/ American Stroke Association. Stroke 2015;46(10):3020-3035

5 Zenteno M, Moscote-Salazar LR, Alvis-Miranda H, Lee A. Use of heparin in neurointervention: a review of the literature. Romanian Neurosurg 2013;20(4):369-374
6 Paniccia R, Priora R, Liotta AA, Abbate R. Platelet function tests: a comparative review. Vasc Health Risk Manag 2015;11:133-148

7 Yeung J, Holinstat M. Newer agents in antiplatelet therapy: a review. J Blood Med 2012;3:33-42

8 Madigan J. Vascular access: guide catheter selection, usage, and compatibility. In: Interventional Neuroradiology. Techniques in Interventional Radiology. London, United Kingdom: Springer; 2014:27-38

9 Velasco A, Buerke B, Stracke CP, et al. Comparison of a balloon guide catheter and a non-balloon guide catheter for mechanical thrombectomy. Radiology 2016;280(1):169-176

10 Turk AS, Frei D, Fiorella D, et al. ADAPT FAST study: a direct aspiration first pass technique for acute stroke thrombectomy. J Neurointerv Surg 2014;6(4):260-264

11 Gonzalez FL, Albuquerque FC, McDougall CG. Neurointerventional Techniques: Tricks of theTrade. New York, NY: Thieme Medical Publishers; 2014

12 Vaidya S, Tozer KR, Chen J. An overview of embolic agents. Semin Intervent Radiol 2008;25(3):204-215

13 Eskioglu E, Burry MV, Mericle RA. Transradial approach for neuroendovascular surgery of intracranial vascular lesions. J Neurosurg 2004;101(5):767-769

14 Moret J, Cognard C, Weill A, Castaings L, Rey A. The "Remodelling Technique" in the treatment of wide neck intracranial aneurysms. Angiographic results and clinical follow-up in 56 cases. Interv Neuroradiol 1997;3(1):21-35

15 Li C, Li Y, Jiang C, Wu Z, Wang Y, Yang X. Stent alone treatment for dissections and dissecting aneurysms involving the basilar artery. J Neurointerv Surg 2015;7(1):50-55

16 Krishna C, Sonig A, Natarajan SK, Siddiqui AH. The expanding realm of endovascular neurosurgery: flow diversion for cerebral aneurysm management. Methodist DeBakey Cardiovasc J 2014;10(4):214-219

17 Nogueira RG, Jadhav AP, Haussen DC, et al; DAWN Trial Investigators. Thrombectomy 6 to 24 hours after stroke with a mismatch between deficit and infarct. $\mathrm{N}$ Engl J Med 2018;378(1):11-21

18 Wong J, Telischak N, Heit J, et al. E-083 Acute Stroke Intervention for Large Vessel Occlusion with Combined Stent Retriever and Suction Thrombectomy (Solumbra Technique): a retrospective analysis of 85 patients. J NeuroInterventional Surg 2016;8(Suppl 1):A86-A86

19 Kurre W, Aguilar-Pérez M, Martinez-Moreno R, Schmid E, Bäzner H, Henkes H. Stent retriever thrombectomy of small caliber intracranial vessels using pREset LITE: safety and efficacy. Clin Neuroradiol 2017;27(3):351-360

20 van Rooij WJ, Sluzewski M, Beute GN. Brain AVM embolization with Onyx. AJNR Am J Neuroradiol 2007;28(1):172-177, discussion 178

21 Schwartz BG, Burstein S, Economides C, Kloner RA, Shavelle DM, Mayeda GS. Review of vascular closure devices. J Invasive Cardiol 2010;22(12):599-607

22 Davis MC, Deveikis JP, Harrigan MR. Clinical presentation, imaging, and management of complications due to neurointerventional procedures. Semin Intervent Radiol 2015;32(2):98-107

23 Kallmes DF, Hanel R, Lopes D, et al. International retrospective study of the pipeline embolization device: a multicenter aneurysm treatment study. AJNR Am J Neuroradiol 2015;36(1):108-115 